\title{
Platform Pricing Structure and Moral Hazard*
}

\author{
Guillaume Roger ${ }^{\dagger} \quad$ Luís Vasconcelos ${ }^{\ddagger}$
}

December 2013

\begin{abstract}
We study pricing by a two-sided platform when it faces moral hazard on the sellers' side. In doing so, we introduce an equilibrium notion of platform reputation in an infinite horizon model. We find that with transaction fees only, the platform cannot eliminate the loss of reputation induced by moral hazard. If registration fees can be levied, moral hazard can be overcome. The registration fee determines the participation threshold of sellers and extracts them, while (lower) transaction fees provide incentives for good behavior. This provides a motivation for platforms to use registration fees in addition to transaction fees.
\end{abstract}

JEL Classification: L11,L12,L14,L81D21,D82.

Keywords: platforms, two-sided markets, reputation, moral hazard, two-part tariff.

\footnotetext{
${ }^{*}$ We would like to thank Juan Carrillo, Nancy Gallini, Iliyan Georgiev, Bob Gibbons, Steffen Hoernig, Bruno Jullien, Gustavo Manso, Jean-Charles Rochet, Richard Schmalensee, and Romain de Nijs for helpful discussions and comments, as well as seminar participants at Caltech, the University of Melbourne, the University of Southern California, the University of New South Wales, the University of Essex, the Nova School of Business and Economics, the Universidade de Évora, and conference attendees at the 2008 North American Summer Meeting of the Econometric Society, the 2008 International Industrial Organization Conference, the 2010 Conference of the European Association for Research in Industrial Economics, the 2010 Annual Meeting of the Association of the Southern European Economic Theorists, and the 2nd Workshop on the Economics of ICTs. Any errors that remain are ours. Luís Vasconcelos gratefully acknowledges financial support from the Fundação para a Ciência e Tecnologia under grant POCI/EGE/58934/2004.

$\dagger$ Visiting Fellow, School of Economics, University of Sydney, Sydney, Australia. E-mail: guillaume.roger72@gmail.com

‡University of Essex, Colchester, UK. E-mail: lvasco@essex.ac.uk
} 


\section{Introduction}

Marketplaces, stock exchanges, and internet-based trading platforms bring together sellers and buyers of all stripes. Typically it is difficult to control the behavior of these traders as they transact not with the platform but directly with each other. Hence opportunistic behavior can reasonably be expected to arise, and evidently does. ${ }^{1}$ While the trading parties are the ones directly injured, this is a problem for the platform as it affects the value of the service it offers, and therefore its ability to extract surplus from traders. That is, platforms face a problem of reputation, which they can govern only indirectly through the behavior of the parties they host.

For concreteness, consider the example of Apple Inc. It acts as a platform by providing a marketplace for applications, the App Store, where third-party developers list their wares for Apple users to purchase. Apple does not carefully audit the quality of these (currently 500,000) products. Apple also cannot directly control the actions of developers, whose lack of effort may result in applications with security flaws, poor performance or that interfere with other software. Developers may even engage in malicious behavior such as phishing or outright fraud. Clearly, these problems may affect the credibility of the App Store, and therefore its ability to sell these applications.

In this paper we analyze how the pricing structure of a platform (like the App Store) affects the prevalence of opportunistic behavior by traders. We show that the use of registration fees (in addition to transaction fees) helps mitigate the moral hazard problem to the point of overcoming it entirely in some instances. Building on the construct of Rochet and Tirole (2003), we study a model of repeated interactions over an infinite horizon. Buyers and sellers need an intermediary (the platform) to transact, which can charge them fees for its intermediation services. To capture the problem of opportunistic behavior, we let sellers pick one of a good and

\footnotetext{
${ }^{1}$ For example, AlibabaScam.com is an independent site dedicated to outing scammers that operate on the popular Alibaba.com. In April 2008, a German court ordered eBay to take preventive measures to guard against the sale of fake Rolex watches.
} 
a bad action, which affects the buyer's payoff directly. The good action is more costly to sellers, but more valuable to buyers-for example developers exert effort to ensure their applications do not compromise computer security. Buyers do not observe the action of their counterparty before the transaction is completed.

At the heart of the paper is the following. Buyers hold some belief that a seller they are matched with takes the good action. This belief is the reputation of the platform. A good reputation is helpful in that the buyers' expected value from a trade increases with reputation, and so does their willingness to trade. Given the fees charged by the platform and its reputation, buyers decide whether to trade. If taking the bad action, sellers are detected ex post with positive probability and may be excluded from the platform. ${ }^{2}$ Hence a cheating seller runs the risk of foregoing future trades. The exact cost of exclusion depends on the fees and the number of participating buyers, as it affects the probability of future transactions. Given fees and the mass of buyers willing to trade, sellers (i) decide whether to trade, and (ii) which action to choose if trading. In equilibrium, given the fees charged by the platform, buyers anticipate sellers' behavior and optimally respond to it and sellers anticipate buyers' behavior and optimally reply to it.

We show that combined with the threat of exclusion, prices turn out to be simple and effective tools to mitigate traders' opportunistic behavior. ${ }^{3}$ Our main result states that with registration fees and transaction fees (a two-part tariff), the moral hazard problem can be

\footnotetext{
${ }^{2}$ Most trading platforms reserve the right to exclude participants if they are harmful to others, as for example eBay, MySpace, Yahoo!Stores, Match.com, GumTree.com.au, or even the more laissez-faire craigslist.com. See their respective Terms and Conditions. This is also the case with the App Store. For example, it is known to have excluded developers and pulled down applications such as a fake driver-license app and "Process Killer", an app able to stop other apps running in the background.

${ }^{3}$ Some platforms may use a broader set of instruments, such as vetting their members, or policing them, as do securities exchanges such as the New York Stock Exchange (NYSE) or the Chicago Mercantile Exchange (CME). For example, the CME regularly audits its own clearing members for financial viability. Furthermore, the NYSE and the National Association of Securities Dealers (NASD) have become a Self-Regulatory Organization (SRO) through their joint enforcement arm called the Financial Industry Regulatory Authority (FINRA). An SRO is able to enact and enforce its own rules, as well as the broader rule of law, and engages in dispute resolution between parties. However no Internet trading site qualifies as an SRO.
} 
entirely overcome in the sense that the platform can implement equilibria where all participating sellers choose the good action. Moreover, the optimal outcome for the platform (in terms of reputation and profit) absent moral hazard can be fully replicated, when the buyers' valuation for the good action is high enough. None of this can be achieved with transaction fees alone, and the latter requires a proper combination of transaction and registration fees.

In this combination, distinct elements of the two-part tariff have different implications for traders' behavior. The registration fees affect only the agents' participation decision (i.e., whether to trade on the platform). While sellers trade off the registration fee with the present value of future trade opportunities when deciding whether to join the platform, that fee is sunk when they decide between the good and the bad action in a given transaction. In contrast, transaction fees affect both the participation decision and the incentive constraint (i.e., whether to behave opportunistically). Increasing transaction fees, for example, decreases the net value of a transaction for a seller, and thus decreases sellers' participation. It also erodes the sellers' present value of future trades and therefore the salience of exclusion as a punishment. Consequently, the incentives to behave opportunistically increase.

When a platform charges transaction fees only, it has an incentive to offer a discount (compared to the case without moral hazard) in order to induce less opportunistic behavior. Sellers choosing the bad action are those with a lower valuation of trading on the platform: for them, exclusion is less costly. By decreasing transaction fees, the platform provides more incentives for active sellers to choose the good action: it increases the present value of future trade, that is, the salience of the punishment. But it also invites lower-valuation sellers who will choose the bad action. So there is always a non-zero measure of sellers who participate and take the bad action. This problem can be easily overcome if the platform can charge registration fees, which are used to keep out low-valuation sellers. With the appropriate registration fee, only high-valuation sellers, who have the incentive to choose the good action, register and trade on the platform. Opportunistic behavior can be entirely overcome. Registration fees are not only 
useful to restore reputation by keeping out unwanted traders, combined with transaction fees they also improve the platform's profit.

Our results are important for the following reasons. First, they show that platforms can address a (potential) moral hazard problem with simple pricing instruments combined with exclusion. In particular, platforms can use registration fees to keep low-valuation traders who are more prone to behave opportunistically out of the platform. This seems to be in line with the Apple example, where small developers pay $\$ 100$ to access the source codes (and keep $70 \%$ of the revenue associated with the sale of their apps). They also suggest that lowering registration fees to increase market share, for example, may have adverse consequences: it attracts low-valuation sellers, which increases the prevalence of opportunistic behavior. Second, our results provide a novel explanation for the diversity of pricing schemes that we observe in reality. Specifically, some platforms (for example, Half.com) charge transaction fees only, while others use both transaction fees and registration fees (such as App Store, Yahoo!Stores or MySpaceMyAds.com). We offer a rationale for the latter. Finally, our results may have policy implications. They show that mark-ups may be highly skewed (as in Bolt and Tieman, 2008) as a response to opportunistic behavior and not just because of demand elasticities. An antitrust policy based on the analysis of markups must take such an effect into account.

A growing literature has been studying optimal pricing by platforms that connect two sides of a market (e.g., Caillaud and Jullien, 2003; Rochet and Tirole, 2003; Armstrong, 2006; Rochet and Tirole, 2006; Hagiu, 2006; Weyl, 2010; Reisinger, 2010). The present paper departs from all these in that we allow for moral hazard on the sellers' side. An important implication is that transaction and registration fees are not equivalent pricing instruments, even under monopoly. This differs from Armstrong (2006) and Rochet and Tirole (2006), where they are interchangeable for a monopoly platform. Equivalence may be void for other reasons, as in Reisinger (2010). There, traders are heterogenous in their probability of trade, so transaction fees affect different types of traders differently, while registration fees have the same impact 
for all traders. Therefore a change in the combination of fees that leaves one type indifferent, may leave another type of seller worse off leading him to abandon the platform. Here agents decide both whether to participate and to act opportunistically. Registration fees differ from transaction fees primarily because they affect these two decisions differently. The former affect only the decision to participate, whereas transaction fees affect both. Registration fees may also differ from transaction fees under platform competition. As highlighted by Armstrong (2006), transaction fees affect the surplus per transaction and therefore the cross-market externality. This influences the intensity of competition for traders between platforms, whereas registration fees remain neutral. In Caillaud and Jullien (2003) and Hagiu (2006), registration fees are used to break bad expectations of one side of the traders on a platform about the other side's participation (also in a setting with platform competition). This is because the impact of registration fees on the agents' payoff is independent of the transaction volume, and therefore of their expectations about participation on the other side. Neither of these two effects are present in out model, as we focus on the case of a monopoly platform.

In this paper registration fees may be used to sort sellers. Damiano and Li (2007) study a situation in which a matchmaker also uses registration fees to sort agents. In that paper matching payoffs are complementary in the agents' types, so the efficient pairing is positively assortative. To maximize revenue, the platform can then create several meeting places, where similar agents on both sides meet and trade. Because the platform does not observe agent's types, it uses registration fees to sort sellers into each meeting place. ${ }^{4}$ As in Damiano and Li (2007), our platform cannot observe traders' types (valuations) and uses registration fees to sort sellers. However the motivation to screen sellers is not payoff complementarities, but moral hazard. Registration fees work well because the incentives that drive the action choice are correlated with the sellers' type; the low-valuation sellers are precisely those who have

\footnotetext{
${ }^{4}$ Halaburda and Piskorski (2011) study the case of a plaform which adds value to agents with low outside option by restricting choice. In their case too, the platform screen agents with registration fees.
} 
incentives to behave opportunistically. So to solve its moral hazard problem, the platform has to address a problem of adverse selection too.

Finally, our paper relates to a growing literature on platform design where the platform may be able to use non-price instruments to affect the quality and behavior of its users. Boudreau and Hagiu (2009) and Hagiu (2009) let the platform directly select the types of traders it allows in order to control the average quality. In Hagiu and Jullien (2010), it may downgrade the quality of the service to some users in order to direct their counterparty to their competitors. As in that literature, we analyze the problem of a platform that attempts to improve the quality of the service it offers. By combining price instruments with exclusion, a non-price instrument, the platform can eliminate opportunistic behavior on the part of sellers, increase the value of its services and improve profits.

The remainder of the paper is organized as follows. In the next section we lay out the model. In Section 3, we analyze the moral hazard problem with transaction fees only. In Section 4, we allow for registration fees in addition to transaction fees. An example is presented in Section 5. Section 6 discusses some robustness issues related to our key findings. Section 7 concludes. All the proofs are in the Appendix.

\section{The Model}

Agents and primitives There is a single platform that offers intermediation services to a continuum of heterogenous sellers and a continuum of heterogenous buyers every period. Sellers and buyers need this intermediation: they cannot meet by themselves. Sellers are indexed by $s \in \mathbb{R}$ and buyers by $b \in \mathbb{R}$ - their type. Sellers are long-lived and buyers live one period only. In each period a new population of buyers with measure one is drawn from a log-concave distribution $G$ with positive density $g .{ }^{5}$ The precise characterization of the population of sellers

\footnotetext{
${ }^{5}$ Assuming short-lived buyers simplifies the analysis and the exposition considerably, but none of our results depend on it. They all remain valid if, similarly to the case of sellers, we assume that a buyer dies with some
} 
and its dynamics will be made below. Time is discrete and indexed by $\tau$.

Actions and payoffs Conditional on patronizing the platform, a seller is randomly matched in each period with a buyer. ${ }^{6}$ Upon a match a seller chooses $(i)$ whether to trade and $(i i)$ if trading, an action $a \in\{l, h\}$. We assume that sellers' decisions are made after they are matched with a buyer for simplicity of exposition. The precise order with which these events occur is immaterial to the analysis and results. Hence the model captures, for example, both the cases of an eBay seller who decides whether to trade and to ship a fake product after being matched with a buyer, and the case of an application developer who first creates the product and then lists it on the App Store. We assume that a seller of type $s$ values a transaction according to

$$
v(s, a)=\left\{\begin{array}{lll}
s+d & \text { if } & a=l \\
s & \text { if } & a=h
\end{array}\right.
$$

where $d>0$, so action $h$ is costly to the seller as compared to action $l$. If matched, a buyer faces the sole decision of whether to trade. A buyer of type $b$ values a transaction with a seller taking action $a$ following

$$
u(b, a)=\left\{\begin{array}{lll}
b & \text { if } & a=l \\
b+y & \text { if } & a=h,
\end{array}\right.
$$

where $y>0$. So a buyer always prefers that the seller take the high-cost action $h$ over the alternative $l$. The parameter $y$ is important in our model. It measures the buyers' sensitivity to the sellers' action and, as we will see, the importance of reputation for the platform. We probability at the end of each period and a fixed measure of new buyers enters the market.

${ }^{6}$ The fact that every seller is matched with a buyer is compatible, for example, with a situation where the population of buyers is larger than that of sellers. Indeed, we make the necessary assumptions below to ensure that this is the case. So, while every seller is matched with a buyer, not every buyer is matched with a seller. However, the main results in this paper do not depend on these particular assumptions on the matching technology and the relative size of the buyers and sellers' populations. They continue to hold if we consider more generally that the probability that a seller be matched with a buyer is less than one and a function of the number of buyers and sellers. Finally, the fact that matching is left random is line with the literature, see Rochet and Tirole (2003) for example. 
assume that $y>d$, so that implementing action $h$ is socially optimal. Whenever a buyer and a seller complete a trade, they must pay transaction fees $t^{b}$ and $t^{s}$, respectively, to the platform. The net payoff to a seller of type $s$ is $v(s, a)-t^{s}$ and to a buyer of type $b$ is $u(b, a)-t^{b}$. Both buyers and sellers get zero when they do not trade.

Information Each side of the market is uninformed as to the other's type upon transacting. In particular, the buyer does not observe the type of the seller, whose choice of action $a$ may depend on his type. Moreover, the buyer does not observe the seller's action before the transaction is completed. Without this information, the buyer's valuation of a trade depends on his expectation (perceived probability) that the seller will choose action $h$. We call this expectation the platform's reputation. It plays an important role in the buyer's decision to trade. In equilibrium, it must be identical to the proportion of sellers who choose action $h$, among those who trade. The platform remains uninformed as to sellers' and buyers' types. However, whenever a seller chooses action $l$, the platform observes it with probability $\alpha<1$ after the transaction is completed. The platform never receives a wrong signal: no signal is received by the platform if a seller chooses action $h$. This signal structure attempts to reflect imperfections in buyer feedback to the platform (e.g., disputes may be settled directly), or the platform's policy of non-systematic arbitration of dispute. ${ }^{7}$ We assume that sellers' actions are not verifiable, or contracting costs are prohibitive compared to the gains from trade. Therefore contracts that are contingent on the sellers' actions are not feasible. When such contracts are feasible there is no role for reputation.

Prices and incentives The platform charges transaction fees to buyers and sellers whenever a transaction is completed; it may also use registration fees to regulate access. Absent some

\footnotetext{
${ }^{7}$ For example, platforms like MySpace and Match.com explicitly state in their "Terms of use Agreement" that they reserve the right, but have no obligation, to become involved in any way with disputes between members, and in disputes between members and other users.
} 
incentive device, a buyer has no reason to believe that any seller will take the high action $h$; that is, the platform's reputation would always be zero. We call on intertemporal incentives and assume a natural form of punishment: if detected, sellers who chose action $l$ are excluded from the platform. That is, they are prevented from trading through the platform forever. Almost all trading platforms or other platforms that offer intermediation services do reserve the right to exclude members whose behavior they deem inappropriate. Apple Inc reserves the right to exclude developers from the App Store (and in fact does so routinely). ${ }^{8}$

Sellers population dynamics With probability $m$ a seller leaves the market ("dies") at the end of each period $\tau$. This probability is exogenous, independent of the seller's behavior and type. Because excluded sellers cannot trade anymore on the platform, we treat them as sellers who leave the market. Hence, in each period sellers may leave the market either because they die or because they are excluded from the platform. Every period a fixed number (measure) $E$ of new sellers, drawn from a $\log$-concave distribution $F$ with everywhere positive density $f$, enter the market. We assume that $E \leq m$. This ensures that in any equilibrium the measure of sellers is smaller than that of buyers, justifying the simplifying assumption on the matching technology that all sellers are matched with a buyer. We further assume that the ratio $[1-F(s)] /[1-F(s-k)], k>0$, is bounded away from zero. ${ }^{9}$ With these entry and exit movements of sellers, the distribution and number (measure) of sellers in the population may vary from one period to the next, although we will focus on stationary distributions.

In this setup we formalize the idea of equilibrium reputation of the platform and transactions, establish existence of an equilibrium, analyze how fees affect that reputation, and address the question of optimal fees. This analysis is performed in a steady-state, where the measure of

\footnotetext{
${ }^{8}$ While exclusion is a natural form of punishment, all the analysis and results in the paper continue to hold if instead the platform requires a seller caught cheating to re-register with the platform. We discuss this issue in more detail in Section 6.

${ }^{9}$ This assumption is used for Lemma 3 only and is satisfied, for example, for the Logistic and the Extreme Value distribution.
} 
sellers and the proportions of sellers who do not trade on the platform, trade and choose action $l$, and trade and choose action $h$ are all time invariant. Buyers and sellers seek to maximize (total discounted) utility, while the platform maximizes total discounted profits. The common discount factor is $\delta<1$.

\section{Transaction Fees and their Shortcomings}

We start by analyzing the case where the platform charges transaction fees only. This allows us to expose the basic mechanics of the model in a simpler setting than with a two-part tariff. Also, this demonstrates the limitations of transaction fees under moral hazard.

\subsection{Equilibrium Reputation and Transactions}

We begin with the characterization of the buyers' trading decisions given the platform's reputation and the transaction fee $t^{b}$ charged to buyers, then turn to the characterization of the sellers' decisions given the behavior on the buyer side and the transaction fee $t^{s}$ charged to sellers. Combining these two enables us to derive the equilibrium reputation and transaction volumes, given the fees charged by the platform.

Buyers' transaction decisions Let $r$ be the reputation of the platform. That is, $r$ is the probability assigned by a buyer that when trading with a randomly drawn seller, the latter chooses action $h$. If matched, a buyer faces the choice of whether to trade. Conditional on being of type $b$, her expected value from the transaction is $r u(b, h)+(1-r) u(b, l)-t^{b}=b+r y-t^{b}$. Hence the buyer trades if and only if $b+r y-t^{b} \geq 0$. Given this, we can define

$$
D^{b}\left(t^{b}, r\right) \equiv 1-G\left(t^{b}-r y\right)
$$


as the proportion of buyers who accept to trade on the platform given the transaction fee $t^{b}$ and $r$ (reputation). We refer to $D^{b}\left(t^{b}, r\right)$ as buyer participation. It can be interpreted as the buyers' demand for transactions on the platform. Following the assumptions on $G(b), D^{b}\left(t^{b}, r\right)$ is continuous, decreasing in $t^{b}$, and increasing in $r$. An immediate consequence of this observation is that reputation is valuable to the platform. All things otherwise equal, the higher the platform's reputation, the higher the measure of buyers willing to trade on it.

Sellers' transaction decisions given buyer participation Fix buyer participation. Let it be $X^{b}$ in any given period. ${ }^{10}$ Upon being matched with a buyer, a seller has two decisions to make: whether to trade and which action $a \in\{l, h\}$ to take. These decisions depend on the seller's payoff from the current transaction, and on the seller's continuation value of trade. When buyer participation is the same in every period, the seller's problem is stationary and the seller's continuation value of trade is also the same in every period. For a seller of type $s$, let $V(s)$ denote the present value of the expected future payoffs at the beginning of a period. $V(s)$ corresponds to the value of the platform to a seller of type $s$ and satisfies the (Bellman) equation

$$
\begin{aligned}
V(s)= & X^{b} \max \left\{0+(1-m) \delta V(s), s+d-t^{s}+(1-\alpha)(1-m) \delta V(s), s-t^{s}+(1-m) \delta V(s)\right\} \\
& +\left(1-X^{b}\right)(1-m) \delta V(s) .
\end{aligned}
$$

With probability $X^{b}$ the seller is matched with a buyer who wishes to trade and faces three options: (i) he does not trade but receives $V(s)$ next period, (ii) he trades and chooses $l$, collects $s+d-t^{s}$ now and receives $V(s)$ with probability $1-\alpha$ next period, (iii) he trades and selects $h$, collects $s-t^{s}$ and receives $V(s)$ next period for sure. With probability $1-X^{b}$ the seller is not matched with a buyer willing to trade. Throughout, a seller dies with probability $m$. Hence

\footnotetext{
${ }^{10}$ Because we are interested in steady-state equilibria where all variables are stationary, it is sufficient to study sellers' optimal behavior when buyer participation is the same in every period.
} 
Lemma 1 A seller of type $s$ accepts to trade if and only if $s+d \geq t^{s}$.

Only sellers with a high-enough valuation trade, whereas the others do not. The traders consist of the sellers to whom the net value of a transaction is positive when they choose action l. Given transaction fee $t^{s}$, the exact proportion of sellers accepting to trade in period $\tau$ depends on the distribution of sellers in that period $F_{\tau}$. Let

$$
D_{\tau}^{s}\left(t^{s}\right) \equiv 1-F_{\tau}\left(t^{s}-d\right)
$$

denote that proportion. $D_{\tau}^{s}\left(t^{s}\right)$ is referred to as seller participation. It is decreasing in $t^{s}$. For expositional convenience, let $s_{l}\left(t^{s}\right) \equiv t^{s}-d$ represent the threshold level of $s$ above which sellers accept to trade given transaction fee $t^{s}$. Then $D_{\tau}^{s}\left(t^{s}\right)=1-F_{\tau}\left(s_{l}\left(t^{s}\right)\right)$.

The sellers' incentive problem is to choose between actions $l$ and $h$. A seller takes the high-cost action $h$ if and only if the immediate gain from cheating on the current transaction, $d$, does not exceed the reduction of his expected continuation value of trade if being excluded from the platform. That is, a seller of type $s$ who trades chooses action $h$ if and only if $d \leq \alpha(1-m) \delta V(s)$. The optimal action is characterized as follows.

Lemma $2 A$ seller of type $s$ who accepts to trade (and expects buyers' participation to be $X^{b}$ in the subsequent periods) chooses action $h$ if and only if

$$
d \leq \alpha \frac{(1-m) \delta}{1-(1-m) \delta} X^{b}\left(s-t^{s}\right)
$$

When condition (4) holds, $s$ and $V(s)$ are sufficiently high so that choosing action $h$ is optimal, and conversely if it fails. We see that sellers' incentives to choose action $h$ depend not only on $t^{s}$ but also on the buyers' participation $X^{b}$. These cross-market effects are a central feature of two-sided markets. ${ }^{11}$ For expositional convenience, let $s_{h}\left(t^{s}, X^{b}\right)$ denote the threshold

\footnotetext{
${ }^{11}$ For example, in Caillaud and Jullien (2003), the sellers' decision to register on the platform depends on the buyers' participation on the platform and vice-versa. Here it is the seller's incentive to choose the high action
} 
value of $s$ above which sellers choose action $h$; it must satisfy $s_{h}\left(t^{s}, X^{b}\right)>s_{l}\left(t^{s}\right)$ for all $t^{s}$ and $X^{b}>0$. Then given $t^{s}$ and $X^{b}>0$, we have three cases:

- $s<s_{l}\left(t^{s}\right)$ sellers do not trade;

- $s \in\left[s_{l}\left(t^{s}\right), s_{h}\left(t^{s}, X^{b}\right)\right)$ sellers trade and choose action $l$; and

- $s \geq s_{h}\left(t^{s}, X^{b}\right)$ sellers trade and choose action $h$.

Equilibrium In equilibrium, buyers' behavior has to be optimal given fees and reputation, sellers' behavior has to be optimal given fees and buyers' participation, and platform reputation has to be consistent with sellers' behavior. The proportion of sellers who trade on the platform and the proportion of sellers who trade and choose action $h$ depends on the distribution of sellers and may evolve over time. This is because some sellers leave the market and others, not necessarily identical to them, enter it. In particular, excluding sellers in a given period whose types are in the interval $\left[s_{l}\left(t^{s}\right), s_{h}\left(t^{s}, X^{b}\right)\right)$ may imply a reduction in the proportion of those types of sellers. Throughout, we focus on steady-state equilibria: the proportions of sellers who do not trade on the platform, trade and choose action $l$, and trade and choose action $h$, are time invariant. Let $N, L$, and $H$ denote, respectively, these proportions. ${ }^{12}$

Definition 1 (Equilibrium transactions and reputation) Given transactions fees $t^{b}$ and $t^{s}$, a steady-state equilibrium is a tuple $\left\langle X^{b *}, N^{*}, L^{*}, H^{*}, S^{*}, r^{*}\right\rangle$ such that:

1. (steady-state conditions) the number of total sellers and the proportions of those sellers who do not trade, trade and choose action $l$, and trade and choose action $h$ remain constant,i.e.,

in a transaction that depends on the buyer's participation on the platform.

${ }^{12}$ Alternatively, we could have focused on situations where the entire distribution of sellers is time invariant. Note, however, that our equilibrium concept encompasses such cases as special. A time invariant distribution of sellers implies time invariant proportions of sellers who do not trade on the platform, trade and choose action $l$, and trade and choose action $h$. 
(a) $E F\left(s_{l}\left(t^{s}\right)\right)=m N^{*} S^{*}$

(b) $E\left[F\left(s_{h}\left(t^{s}, X^{b *}\right)\right)-F\left(s_{l}\left(t^{s}\right)\right)\right]=\left[m+(1-m) X^{b *} \alpha\right] L^{*} S^{*}$

(c) $E\left[1-F\left(s_{h}\left(t^{s}, X^{b *}\right)\right)\right]=m H^{*} S^{*}$

(d) $N^{*}+L^{*}+H^{*}=1$;

2. (buyer participation is consistent with $r^{*}$ and $\left.t^{b}\right) X^{b *}=D^{b}\left(t^{b}, r^{*}\right)$ and

3. (reputation is consistent with seller behavior) $r^{*}=\frac{H^{*}}{L^{*}+H^{*}}$.

Equilibrium transaction volumes and reputation are then characterized by these six conditions. The first four are necessary for the proportions $N, L$, and $H$ to be stationary, well defined, and consistent with seller optimal behavior given $t^{s}$ and buyer participation $X^{b *}$; they also determine the (endogenous) measure of active sellers $\left(S^{*}\right)$. Stationarity follows from conditions 1.(a)-1.(c), which require the number of new sellers entering the market to be identical to the number of sellers exiting, (a) among those sellers who do not trade on the platform, (b) those who trade and cheat, and (c) those who trade and do not cheat. The fifth condition dictates that buyers' transaction decisions be optimal given transaction fee $t^{b}$ and platform reputation. The last condition states that platform reputation be consistent with the sellers' optimal behavior regarding action $a$. The first result establishes the existence of an equilibrium and qualifies equilibrium reputation under transaction fees.

Proposition 1 For each $\left(t^{s}, t^{b}\right)$ an equilibrium exists. In any equilibrium $r^{*} \in(0,1)$.

Irrespective of the transaction fees charged by the platform to sellers and buyers, the equilibrium reputation of the platform is always strictly smaller than one. There is always a positive measure of sellers who trade on the platform and choose action $l$. These are the sellers in the interval $\left[s_{l}\left(t^{s}\right), s_{h}\left(t^{s}, X^{b *}\right)\right)$. 
Although no equilibrium in which reputation is equal to one exists, the level of transaction fees does affect the equilibrium reputation. ${ }^{13}$ A higher fee $t^{b}$ for buyers implies that fewer of them trade when matched with a seller. This decreases the continuation value $V(s)$, which withers the punishment. The incentive to choose the more costly action $h$ is therefore less powerful and in equilibrium, the reputation of the platform drops. Altering the sellers fee $t^{s}$ has a slightly more intricate impact. A higher fee affects the sellers' participation - this is the participation effect. But a higher transaction fee also depresses the continuation value $V(s)$, so fewer sellers choose action $h$ - this is the incentive effect. Thus a higher $t^{s}$ implies fewer sellers with low valuation $s$ (who would choose action $l$ ), but more cheating among higher valuation sellers. Whether increasing $t^{s}$ has a positive or negative effect on reputation depends on the magnitude of each of these two effects. In our model, the second effect is dominant, implying that equilibrium reputation decreases with $t^{s} .{ }^{14}$

Reputational concerns influence the platform's choice of $t^{s}$ and $t^{b}$ in the following sense. In a standard model, transaction fees affect the profit per transaction $\left(t^{s}+t^{b}\right)$ and the number of transactions $D^{b} D^{s} S$. Without moral hazard, volumes respond directly to prices $t^{b}$ and $t^{s}$ (participation decision). In our model, there is an additional channel: both $t^{s}$ and $t^{b}$ also affect the equilibrium reputation, which in turn modifies buyer participation and the number of total sellers in the population. Optimal transaction fees, which maximize the platform's profit

$$
\pi=\frac{\delta}{1-\delta} D^{b} D^{s} S\left(t^{s}+t^{b}\right)
$$

where $D^{b}$ and $D^{s}$ are consistent with the equilibrium, balance all these effects. When the parameter $y$ is high (i.e. buyers are sensitive to reputation), the platform has the incentive to ensure a high reputation. Using transaction fees only, it faces two limitations. First, as

\footnotetext{
${ }^{13}$ In general, one cannot rule out the possibility of multiple equilibria. In what follows, we focus on stable equilibria. We define stable equilibrium as an equilibrium in which, following a small perturbation in the level of reputation, a convergence back to the equilibrium occurs.

${ }^{14}$ For a formal statement and a proof of this result see Roger and Vasconcelos (2011).
} 
stated in Proposition 1, the platform is not able to achieve a reputation of one, although it may be optimal (see benchmark case below). Second, to achieve a high reputation, the platform has to charge sellers low transaction fees, which erodes profits. Before continuing to the analysis of the effect of registration fees on equilibrium outcomes and platform's profits, we analyze a benchmark where the platform can directly control sellers' actions and, therefore, its reputation. This benchmark further illustrates the limitations of transaction fees, as it shows that a reputation of one may be optimal when the platform can directly control sellers' behavior. Also, it helps highlight the role of registration fees. It will serve a reference against which to compare equilibrium outcomes when the platform charges such fees.

\subsection{A Benchmark Case With no Moral Hazard}

Suppose that the platform can select directly the reputation $r$ it wants, in addition to the transaction fees $\left(t^{s}, t^{b}\right)$ it charges. That is, suppose that the platform observes sellers' types and for each seller is able to $(i)$ impose the seller's action $a$ if trading and (ii) prevent a seller from trading. The only thing the platform cannot do is force a seller to trade. ${ }^{15}$ In this setting there is no moral hazard in the sense that the platform can dictate the action of each seller who trades.

Since the sellers who eventually choose action $l$ have the consent of the platform, no exclusion occurs. Thus, the relevant steady-state distribution of sellers is $F$ and their number $S=E / m$. Moreover, since the platform's profit is increasing in the number of transactions (of course, provided $t^{s}+t^{b}>0$ ), given a transaction fee $t^{s}$ it is optimal for the platform to allow all sellers with a valuation $s>t^{s}$ to trade and impose that they choose action $h$. The platform's problem is then to choose the number of low action sellers that it will permit to trade. Let $X^{s}$ denote the proportion of sellers who trade and $r$ the reputation. Reputation must satisfy

\footnotetext{
${ }^{15}$ This is reminiscent of Hagiu (2009), where low-value traders may be excluded.
} 
$r=\left(1-F\left(t^{s}\right)\right) / X^{s}$, which necessarily implies that $X^{s}=\left(1-F\left(t^{s}\right)\right) / r$. We can write the platform's problem as

$$
\max _{t^{s}, t^{b}, r} \Pi\left(t^{s}, t^{b}, r\right)=(E / m)\left[1-G\left(t^{b}-r y\right)\right]\left[\frac{1-F\left(t^{s}\right)}{r}\right]\left(t^{s}+t^{b}\right)
$$

subject to

$$
\frac{1-F\left(t^{s}\right)}{1-F\left(t^{s}-d\right)} \leq r \leq 1
$$

and claim the following.

Lemma 3 For $y$ sufficiently large, the solution of the above problem involves $r=1$ and transaction fees such that $t^{s}+t^{b}=\left[1-F\left(t^{s}\right)\right] / f\left(t^{s}\right)=\left[1-G\left(t^{b}-y\right)\right] / g\left(t^{b}-y\right)$.

The trade-off involves increasing reputation, which buyers value because $y$ is high, at the expense of the number of sellers, who pay $t^{s}$ - even if they choose action $l$. But when $y$ is large enough, the former effect dominates and leads to a corner solution: it is worthwhile dropping all the sellers that choose action $l$. Importantly, when $y$ is large the platform can achieve strictly higher profits when it is able to choose reputation directly than when it is not - the case studied above. As a preview of forthcoming results, if the platform can replicate this allocation by charging a registration fee to sellers, it will necessarily increase its profits relative to the case where it uses only transaction fees.

\section{Registration Fees and Moral Hazard}

As evidenced in the preceding analysis, transaction fees alone are not sufficient to overcome moral hazard. Yet we are about to show that only one additional instrument can be sufficient to do so. Throughout this section we consider the case where the platform may charge registration 
fees to sellers in addition to transaction fees. ${ }^{16}$ Let $T^{s}$ denote the registration fee for sellers. These are paid only once in order to access the platform (like a lifetime membership). Only registered sellers may use the platform.

\subsection{Agents' Decisions and Equilibrium with Registration}

Since buyers do not have to register to trade, the buyers' problem depends only on transaction fee $t^{b}$ and reputation $r$. So the decision rules are identical to those presented in Section 3. Buyer participation is then given by $D^{b}$ as defined by (1).

For registered sellers, the registration fee has no direct bearing on their decisions whether to accept a trade and which action to select. Therefore $V(s)$ is also not affected directly by the registration fee $T^{s} \cdot{ }^{17}$ As before, given transaction fee $t^{s}$ and buyer participation $X^{b}>0$, if registered, sellers of type (i) $s<s_{l}\left(t^{s}\right)$ never trade, (ii) $s \in\left[s_{l}\left(t^{s}\right), s_{h}\left(t^{s}, X^{b}\right)\right)$ trade and choose action $l$, and (iii) $s \geq s_{h}\left(t^{s}, X^{b}\right)$ trade and choose action $h$. Using this, the solution to the Bellman equation (2) reads

$$
V(s)=\left\{\begin{array}{lll}
0 & \text { if } \quad s<s_{l}\left(t^{s}\right) \\
\frac{X^{b}}{1-\delta(1-m)\left[1-\alpha X^{b}\right]}\left[s+d-t^{s}\right] & \text { if } \quad s_{l}\left(t^{s}\right) \leq s<s_{h}\left(t^{s}, X^{b}\right) \\
\frac{X^{b}}{1-\delta(1-m)}\left[s-t^{s}\right] & \text { if } \quad s_{h}\left(t^{s}, X^{b}\right) \leq s
\end{array}\right.
$$

$V(s)$ is continuous and increasing in $s$. Naturally a seller of type $s$ registers if and only if $V(s)-T^{s} \geq 0$. Let $s_{R}\left(t^{s}, T^{s}, X^{b}\right)$ denote the value of $s$ such that this latter condition just binds, when sellers' fees are $t^{s}, T^{s}>0$ and buyer's participation is $X^{b}>0$. For expositional

\footnotetext{
${ }^{16}$ Using registration fees on the buyer side plays no role on the set of equilibrium outcomes. This is because there is no moral hazard on the buyers' side. A proof of this result can be found in Roger and Vasconcelos (2011). Thus, the platform cannot do any better by charging a registration fee to buyers. This is in line with Rochet and Tirole (2006) and Armstrong (2006), who abstract from the issue of moral hazard. In both cases, transaction fees and registration fees are equivalent pricing instruments for a monopoly platform.

${ }^{17}$ It may have an influence through the equilibrium reputation of the platform, which at this point we take as given.
} 
convenience, let $s_{R}\left(t^{s}, 0, X^{b}\right)=s_{l}\left(t^{s}\right)$. Clearly, $s_{R}\left(t^{s}, T^{s}, X^{b}\right) \geq s_{l}\left(t^{s}\right)$ when $T^{s} \geq 0$. Without loss of generality, hereinafter we focus on instances where $T^{s}$ is non-negative, thus sellers of type $s \geq s_{R}\left(t^{s}, T^{s}, X^{b}\right)$ register with the platform and trade, whereas the others do not. ${ }^{18}$ For buyers, the relevant sellers are those who are registered and trade. Hence, redefining seller participation in period $\tau$ as the proportion of sellers who register and trade, it is now given by

$$
D_{\tau}^{s}\left(t^{s}, T^{s}, X^{b}\right) \equiv 1-F_{\tau}\left(s_{R}\left(t^{s}, T^{s}, X^{b}\right)\right) .
$$

Equilibrium With these registration fees, the sellers' participation decision depends not only on transaction fees $t^{s}$, but also on the registration fee $T^{s}$ and on buyers' participation. This is because participation requires registration, and the value of being registered depends on the measure of agents on the other side of the market. ${ }^{19}$ Accordingly, the definition of an equilibrium needs to be adjusted.

Definition 2 (Equilibrium with registration) Given transaction fees $t^{b}$ and $t^{s}$ and registration fees $T^{s}$, an equilibrium is a tuple $\left\langle X^{b *}, N^{*}, L^{*}, H^{*}, S^{*}, r^{*}\right\rangle$ such that:

1. (steady-state conditions) the number of total sellers and the proportions of those sellers who do not trade, trade and choose action $l$, and trade and choose action $h$ remain constant,i.e.,

(a) $E F\left(s_{R}\left(t^{s}, T^{s}, X^{b *}\right)\right)=m N^{*} S^{*}$

(b) $E\left[F\left(\max \left\{s_{R}\left(t^{s}, T^{s}, X^{b *}\right), s_{h}\left(t^{s}, X^{b *}\right)\right\}\right)-F\left(s_{R}\left(t^{s}, T^{s}, X^{b *}\right)\right)\right]=\left(m+(1-m) X^{b *} \alpha\right) L^{*} S^{*}$

(c) $\left.E\left[1-F\left(\max \left\{s_{R}\left(t^{s}, T^{s}, X^{b *}\right), s_{h}\left(t^{s}, X^{b *}\right)\right\}\right)\right)\right]=m H^{*} S^{*}$

\footnotetext{
${ }^{18}$ It is never optimal for the platform to set a negative registration fee $T^{s}$. That would mean to subsidize registration of sellers who do not trade, which would not increase seller participation and consequently would not increase the platform's profit.

${ }^{19}$ In contrast to transaction fees, where only the seller's incentive problem is directly dependent on the buyers' participation.
} 
(d) $N^{*}+L^{*}+H^{*}=1$;

2. (buyer participation and registration is consistent with $r^{*}$ and $\left.t^{b}\right) X^{b *}=D^{b}\left(t^{b}, r^{*}\right)$

3. (reputation is consistent with seller behavior) $r^{*}=\frac{H^{*}}{L^{*}+H^{*}}$.

The definition remains conceptually the same as before. The main difference is that with registration fees, the definition of equilibrium must reflect the fact that only those sellers who register may trade on the platform. Proposition 2 is the counterpart of Proposition 1.

Proposition 2 For each $\left(t^{b}, t^{s}, T^{s}\right)$ there exists an equilibrium.

This result differs from Proposition 1 because when agents have to register to trade on the platform, the equilibrium reputation is not necessarily strictly lower than one. Now we are ready to turn to the main result of the paper.

\subsection{The Role of Registration Fees}

We first note that the equilibrium reputation is increasing in $T^{s}$; registration fees always assist in improving the platform's reputation. The registration fee reduces seller participation (those with a lower type opt out), while not affecting their incentives with respect to the action $a .^{20}$ Since the low-type sellers are precisely those with the incentive to choose action $l$, an increase in registration fees reduces the proportion of cheaters among sellers who trade, thus increasing reputation. That is, the registration fee works as a way of selecting the sellers that trade on the platform. This selection of sellers can be taken to the extreme, so that only those sellers who choose action $h$ if trading, end up registering and trading on the platform. Then

Proposition 3 Given transaction fees $t^{s}$ and $t^{b}$, there exists a registration fee $T^{s}$ such that in equilibrium the platform's reputation is one.

\footnotetext{
${ }^{20}$ For a proof of this result see Roger and Vasconcelos (2011).
} 
By choosing sufficiently high registration fees for sellers, the platform can achieve a reputation of one: no seller chooses action $l$. That is, moral hazard can be entirely overcome. This is never feasible with transaction fees only; in that case the equilibrium reputation is always strictly lower than one. A high reputation is especially valuable when $y$ is large (as shown in the benchmark (Lemma 3)) because then inducing the high action $h$ generates a higher social surplus, some of which accrues to the platform. So it is the standard profit motive that leads the intermediary to seek a high reputation. It is then a matter of finding appropriate transfers to implement this action $h$. This is the object of the next proposition.

Proposition 4 Suppose that it is optimal for the platform to implement a reputation of one in the benchmark case (e.g., $y$ is large enough). Then, there exist fees $\left(t^{s}, t^{b}, T^{s}\right)$ that generate the same allocation as the optimal allocation.

Registration fees can be so helpful as to implement the optimal allocation defined in the benchmark case. In other words, there exist fees that generate the same buyer participation, the same seller participation, and the same profits as those achieved in the benchmark case when buyers value action $h$ sufficiently highly. The reason turns out to be remarkably simple and widely applicable. Transaction fees affect both the sellers' participation and their incentives (the choice of action $a$ ), while registration fees influence the participation decision only. This allows the platform to appropriately combine transaction and registration fees to provide high incentives for sellers to choose action $h$ conditional on participation, and simultaneously to keep low-type sellers out of the platform. With the appropriate mix of fees, the marginal participating seller never takes action $l$, and is made to correspond to the marginal seller absent moral hazard.

We conclude this section with two observations. First, registration fees in this paper are different to posting a bond. This is because registration fees do not directly influence the sellers' behavior. Once paid, they are sunk and a seller's decision to take the low action $l$ is independent 
of $T^{s} \cdot{ }^{21}$ Second, from the formulation of the reputation one can see that it depends positively on $\alpha$. With better monitoring technology, the cost of the low action increases. However, the results that with registration fees the platform can fully eliminate opportunistic behavior and replicate the benchmark allocation without moral hazard do not depend on the precise value of $\alpha$. They only requires that $\alpha>0$. The reason is that even if $\alpha$ is very low, there always exists a combination of registration and transaction fees that implements reputation one and, in particular, the allocation in the benchmark. Of course if $\alpha=0$ reputation is always zero.

\section{An Example}

To further illustrate our results we present a numerical example where we compute optimal fees, platform profits, and platform reputation under transaction fees only and when the platform can charge both types of fees. We also compute social welfare under both pricing regimes. Here total welfare is the sum of the value generated by the transactions that are completed. ${ }^{22}$ Buyers and sellers are drawn from logistic distributions. We fix the parameters $\delta, \alpha, m$, and $d$ and let $y$ vary. Recall that $y$ parameterizes buyers' sensitivity to sellers' action, and therefore

\footnotetext{
${ }^{21}$ This differs from Shapiro (1983), for example, who also studies an intertemporal setting where buyers cannot observe quality prior to purchase. In Shapiro (1983) firm reputation is acquired via the production of quality goods, which are sold below cost in the initial period. This initial investment is recovered over time through a price premium, which provides incentive to continue producing high quality. The free-entry condition implies that future profit flows equal the initial investment in reputation. This initial investment thus works like posting a bond. Here the zero profit condition holds only for the marginal seller; for all the other sellers, expected profit exceeds the initial investment (the registration fee). That is, registration fees affect the platform's reputation not because they work like posting a bond, but through sellers' selection.

${ }^{22}$ Computing welfare is more involved than at first glance: it depends on the number of transactions, the types of sellers and buyers who trade, and the actions chosen by sellers in each transaction. A transaction generates $u(b, l)+v(s, l)=s+d+b$ if the seller chooses action $l$ and $u(b, h)+v(s, h)=s+b+y$ if the seller chooses action $h$. Therefore, total welfare depends on the precise distribution of sellers. Our notion of equilibrium requires only that the proportion of sellers who do not trade on the platform, trade and choose action $l$, and trade and choose action $h$ be time invariant. We focus on equilibria where the distribution of sellers is time invariant. In such equilibria this distribution is uniquely identified. Note also that any equilibrium in this paper can be obtained with a time invariant distribution of sellers. Moreover, for each equilibrium, such a distribution is unique. If $\widehat{f}$ denotes the equilibrium distribution of sellers, total welfare when the platform charges only transaction fees is
}

$$
\int_{s_{l}}^{s_{h}} \int_{t^{b}-r y}^{\infty} S(s+d+b) g(b) \widehat{f}(s) d g d s+\int_{s_{h}}^{\infty} \int_{t^{b}-r y}^{\infty} S(s+b+y) g(b) \widehat{f}(s) d g d s .
$$


to reputation. By varying $y$, we can capture the importance of registration fees as buyers become more sensitive to reputation, and consequently reputation becomes more important to the platform. Table 1 shows the results for several selected values of $y$.

\begin{tabular}{|c|c|c|c|c|c|c|c|c|c|c|c|}
\hline \multirow[b]{3}{*}{$y$} & \multicolumn{5}{|c|}{ Transaction Fees Only } & \multicolumn{6}{|c|}{ Transaction and Registration Fees } \\
\hline & \multicolumn{2}{|c|}{ Optimal Fees } & \multirow[b]{2}{*}{$r^{*}$} & \multirow[b]{2}{*}{ Profit } & \multirow[b]{2}{*}{ Welfare } & \multicolumn{3}{|c|}{ Optimal Fees } & \multirow[b]{2}{*}{$r^{*}$} & \multirow[b]{2}{*}{ Profit } & \multirow[b]{2}{*}{ Welfare } \\
\hline & $t^{b}$ & $t^{s}$ & & & & $t^{b}$ & $t^{s}$ & $T^{s}$ & & & \\
\hline 0.50 & 0.89 & 0.63 & 0.82 & 19.80 & 53.01 & 1.04 & 0.12 & 1.21 & 1 & 21.42 & 55.18 \\
\hline 1 & 1.14 & 0.42 & 0.85 & 25.97 & 69.60 & 1.35 & 0.06 & 0.96 & 1 & 29.05 & 73.04 \\
\hline 1.5 & 1.44 & 0.21 & 0.88 & 33.95 & 90.02 & 1.67 & -0.28 & 1.61 & 1 & 38.53 & 94.42 \\
\hline 2 & 1.79 & 0.001 & 0.9 & 43.97 & 113.49 & 2 & -0.37 & 1.46 & 1 & 50 & 119.31 \\
\hline 2.5 & 2.02 & 0.001 & 0.91 & 55.83 & 135.94 & 2.34 & -0.42 & 1.09 & 1 & 63.55 & 147.58 \\
\hline 3 & 2.32 & 0.001 & 0.92 & 69.04 & 157.60 & 2.68 & -0.54 & 1.01 & 1 & 79.21 & 178.98 \\
\hline
\end{tabular}

Table 1: Optimal fees, equilibrium reputation, profits and welfare when $\delta=0.97, \alpha=0.6$, $m=0.1, E=10$ and $d=0.5$. Values rounded to the second decimal place.

The example confirms that platforms may find it optimal to choose a price structure that completely discourages sellers to cheat. It also illustrates that seeking a reputation of one may be optimal even when $y$ is very close to $d$. The more sensitive buyers are to reputation (i.e. the higher $y$ ), the more essential are the registration fees. The difference between the transaction fees charged to buyers and the transaction fees charged to sellers increases with $y$. This suggests that in the presence of moral hazard on the seller's side, one should expect prices to be (more) skewed toward the buyer side (see Bolt and Tieman, 2008). Note that reputation increases If the platform also charges registration fees, total welfare is similar if $s_{R}<s_{h}$. Simply replace $s_{l}$ in the above expression with $s_{R}$. If $s_{R} \geq s_{h}$, then the expression for the total welfare collapses to

$$
\int_{s_{R}}^{\infty} \int_{t^{b}-y}^{\infty} S(s+b+y) g(b) \widehat{f}(s) d g d s
$$


with $y$ even when the platform charges only transaction fees. ${ }^{23}$

The transaction fees charged to sellers when the platform uses registration fees are lower than those when it does not, and conversely for the buyers'. When the platform charges registration fees to sellers, it needs not use transaction fees to extract surplus, and reducing transaction fees improves incentives (increases reputation). On the buyers' side, fees must be kept low in the absence of registration fees to encourage participation and preserve reputation. When buyers pay a low transaction fee, more buyers trade on the platform, decreasing the sellers' incentive to cheat. But when the platform can use registration fees, it has a substitute tool to keep reputation high. As a consequence, the platform can increase the transaction fee charged to buyers in order to extract more surplus from them.

Not only are both platform profit and social welfare higher under the two-part tariff, the difference in profit and welfare under the two regimes increases with $y$. That is, introducing registration fees is not only privately optimal for the platform, it is also socially efficient. The argument for this is subtle. Total welfare depends on $(i)$ the surplus generated per transaction (quality of transaction) and ( $i i)$ the total number of transactions. With registration fees reputation increases, which implies that the average surplus per transaction (quality) increases. The total number of transactions depends on the number of active buyers and sellers. With registration fees reputation is higher, which encourages buyers to participate, but the platform's response is to increase the buyers' transaction fee. This second effect curtails buyers' participation and is the dominant effect. Introducing registration fees decreases the sellers' participation rate. But it enlarges the pool of active sellers because fewer (or even no) sellers are excluded. Overall, more sellers are active and this more than compensates the loss of buyers. So in this example, more transactions are entered into and each generates a larger surplus.

\footnotetext{
${ }^{23}$ Observe that the optimal price structure depends on the specific distributions of buyers and sellers. In particular, if those distributions are such that the price elasticity of the demand on the buyer side is sufficiently higher than that on the seller side, the platform will optimally charge lower fees to buyers than to sellers. This may explain why some platforms charge lower (or no) fees to buyers even in the presence of moral hazard on the sellers' side.
} 
These mechanics generate negative transaction fees for the sellers - an extreme skewness. Negative transaction fees are not uncommon in two-sided markets, as, for example, credit cards featuring loyalty programs, or free parking at shopping malls. Nonetheless, one may object to negative fees in that they potentially provide a seller and a buyer incentives to collude to collect the payment. Notice first that this could never be the case in the example because the sum $t^{s}+t^{b}$ is always positive (a net payment to the platform). Second, buyers and sellers meet only once per period in this model; that is, it is enough to control the matching technology to preempt this form of collusion. Furthermore, in our model, transaction fees need not be negative for reputation to be one (see Proposition 3).

\section{Discussion}

Our model follows that of Rochet and Tirole (2003), where agents derive usage benefits only. In contrast Armstrong (2006) focuses on heterogenous participation benefits. Even if we let agents be heterogenous in both usage and participation benefits, the role of registration fees that we highlight in this paper remains. With usage and participation benefits, sellers are characterized by valuations along these two dimensions. As before, high-valuation sellers do not take the low action. These now include sellers with low transaction benefits but high-enough participation benefits (which are substitutable). As in our model, registration fees select the high-valuation sellers only.

Throughout the paper we assume that a consumer's benefit of a good action $(y)$ is independent of the buyer's type $(b)$. More generally, they may be linked, i.e., $y \equiv y(b)$. One appealing possibility is that $y$ increases with $b$ : high-valuation buyers also care more about not being cheated. All the results in the paper continue to hold in this case. ${ }^{24}$ The exception is Lemma 3, that states conditions under which a reputation of one is optimal in the benchmark

\footnotetext{
${ }^{24}$ To be rigorous, it is required that $y$ be continuous in $b$.
} 
case without moral hazard. If $y$ increases with $b$, the sufficient condition is no longer that $y$ be sufficiently large, but that $y$ be sufficiently large relative to $y^{\prime}(b)$. This ensures that buyer participation is sufficiently sensitive to the reputation of the platform, leading the platform to choose a high reputation even if that implies a lower volume of transactions.

Similarly we assume that a seller's cost $d$ of taking the good action is independent of the seller's type $s$. It is easy to see that if $d$ decreases with $s$ all our results hold. However, if $d$ increases with $s$ (the cost of the good action is higher for higher-valuation sellers), there may exist equilibria with zero reputation. In some cases only these equilibria may exist. The threat of exclusion is insufficient to induce even the high-valuation sellers to choose the good action: they gain more from trading on the platform, but even more from cheating. Of course, the use of registration fees when there are only equilibria with zero reputation is ineffective. All sellers choose the low action, so there is not point in using registration fees to keep low-valuation sellers out. However, if equilibria with positive reputation exist, registration fees are useful, as in this paper.

We let registration fees be paid once only. In many practical instances registration may have to be renewed, i.e. paid at regular intervals. In that case, registration fees do affect the sellers' continuation value and therefore do change their incentive with respect to the action choice. In other words, registration fees have both a selection effect and an incentive effect. In the extreme, if they are paid every period they act exactly like transaction fees and the benefit of registration disappears. As the interval increases, the incentive effect decreases relative to the selection effect. In these intermediate cases, registration fees do help in sorting sellers. In the limit, which is our case, the incentive effect vanishes. ${ }^{25}$

There may exist other means to deal with moral hazard, such as ex post fines, screening of members, legal remedies, and so forth. The mechanism highlighted in this paper has the

\footnotetext{
${ }^{25}$ Registration fees act like transaction fees when registration has to be renewed every period because of the simplifying assumption that a seller can trade at most once per period. If multiple transactions are possible in the same period, registration fees differ again from transaction fees even if they have to be paid every period.
} 
advantage of being simple, both conceptually and in terms of implementation. It may also be conceivable to let transaction fees vary with a player's history. Without registration fees this can never yield a reputation of one. One could also conceive of asking a seller caught deviating to re-register (pay another fee, which may be different from the initial registration fee) to be allowed back on the platform in lieu of exclusion. At a minimum this requires some information about sellers' behavior; that is, it requires that the platform be able to keep track of the sellers otherwise fees cannot be made contingent on behavior. The sellers' response to such a fee depends on its value. If the fee is lower than $d /(\alpha(1-m) \delta)$ it fails to provide any incentive. Even the high-valuation sellers prefer taking the low action, be caught and re-register than choosing the high action. When the fee is larger than $d /(\alpha(1-m) \delta)$, all the analysis and results in the paper hold. Low-valuation sellers do not trade on the platform. Intermediatevaluation sellers trade, choose the low action and never register again if caught; it is not worth the cost. High-valuation sellers prefer to choose the high action instead of facing the risk of having to re-register to trade again. Thus, such a fee has the exact same effect as excluding sellers who cheat. Seller exclusion and re-registration fees are perfectly substitute mechanisms for the platform.

\section{Conclusion}

In this paper we study pricing strategies available to a monopoly platform in the face of moral hazard on one side of the (two-sided) market. If the platform can use transaction fees only, it can alleviate, but not overcome, the moral hazard problem. This requires some distortion

of its fees relative to the moral hazard-free environment. If registration fees are possible, the platform is able to overcome the moral hazard problem and increase profits. Registration fees allow the platform to select higher valuation sellers with lower incentives to cheat. Improving on the moral hazard problem is important as it increases surplus extraction from both sides. 
The mechanism we suggest is simple and easily implementable. It is also quite robust to perturbations of the assumptions, and importantly, not reliant on the precision of the information $(\alpha)$. Hence we are comfortable drawing some implications for business strategies and antitrust policy. The question of platform competition under moral hazard is left open for future research.

\section{Appendix}

Proof of Lemma 1. First the only if part. Suppose that $s+d<t^{s}$. This implies that also $s<t^{s}$. Since $V(s)$ is non-negative because the seller can always choose not to trade at any given match, it is clear from direct inspection of each of the seller's payoffs inside the curly brackets on the right-hand side of (2) that not trading dominates both trading and choosing action $l$ and trading and choosing action $h$.

Now the if part. Suppose that $s+d \geq t^{s}$. If it is also the case that $s \geq t^{s}$, then regardless of $V(s)$ a matched seller of type $s$ is better off trading and choosing action $h$ than not trading at all-the third term inside the curly brackets in (2) is greater than the first. So in this case trading and choosing action $h$ clearly dominates not trading. If instead $s<t^{s}$, the opposite occurs, i.e., to trade and choose action $h$ is dominated by not to trade at all. As a consequence, (2) collapses into $V(s)=X^{b} \max \left\{0+(1-m) \delta V(s), s+d-t^{s}+(1-\alpha)(1-m) \delta V(s)\right\}+\left(1-X^{b}\right)(1-m) \delta V(s)$. Because $s+d-t^{s} \geq 0$, the solution to this equation is $V(s)=X^{b}\left[s+d-t^{s}\right] /\left[1-(1-m) \delta\left(1-\alpha X^{b}\right)\right]$. Note that this solution satisfies $\delta V(s) \leq s+d-t^{s}+(1-\alpha) \delta V(s)$, which means precisely that for a matched seller not trading is worse than trading and choosing action $l$.

Proof of Lemma 2. As a preliminary fact note that the right-hand side of (4) can be written as $\alpha(1-m) \delta V^{N D}(s)$, where $V^{N D}(s)=[1 /(1-(1-m) \delta)] X^{b}\left[s-t^{s}\right]$ corresponds to the present value of the expected payoffs to a seller of type $s$ who trades on the platform and never cheats. 
We now prove the result in the lemma.

First the only if part. We prove the equivalent statement that if condition (4) is not satisfied, then the seller's optimal decision when matched is not to choose action $h$. Suppose that condition (4) is not satisfied. This means that the seller is better off deviating once and then, if not excluded from the platform, choosing the high action forever. Since the problem is stationary, the seller's optimal decision given a match must always be the same. Thus (always) choosing action $h$ given a match is not optimal.

Now the if part. Suppose that (4) holds. Since the right-hand side of (4) is given by $\alpha(1-m) \delta V^{N D}(s)$ and by definition $V^{N D}(s) \leq V(s)$, this implies that $d \leq \alpha(1-m) \delta V^{N D}(s) \leq$ $\alpha(1-m) \delta V(s)$ and therefore taking the low action cannot be optimal to the seller.

Proof of Proposition 1. Consider an arbitrary pair of fees $\left(t^{b}, t^{s}\right)$. Fix $r \in[0,1]$. Let $X^{b}=D^{b}\left(t^{b}, r\right)$ so that point 2 of Definition 1 is satisfied. Let $x_{l}=s_{l}\left(t^{s}\right)$ and $x_{h}=s_{h}\left(t^{s}, X^{b}\right)$.

Finally, let

$$
\begin{aligned}
N & =\frac{\left[m+(1-m) X^{b} \alpha\right] F\left(x_{l}\right)}{m+(1-m) X^{b} \alpha\left[1-F\left(x_{h}\right)+F\left(x_{l}\right)\right]} \\
L & =\frac{m\left[F\left(x_{h}\right)-F\left(x_{l}\right)\right]}{m+(1-m) X^{b} \alpha\left[1-F\left(x_{h}\right)+F\left(x_{l}\right)\right]} \\
H & =\frac{\left[m+(1-m) X^{b} \alpha\right]\left[1-F\left(x_{h}\right)\right]}{m+(1-m) X^{b} \alpha\left[1-F\left(x_{h}\right)+F\left(x_{l}\right)\right]} \\
S & =E\left\{\frac{1}{m}-\frac{(1-m) X^{b} \alpha\left[F\left(x_{h}\right)-F\left(x_{l}\right)\right]}{m\left(m+(1-m) X^{b} \alpha\right)}\right\}
\end{aligned}
$$

Given $X^{b}, x_{l}$, and $x_{h}$, these values of $N, L, H$, and $S$ constitute the unique solution to the system of equations defined by the set of conditions in point 1 of Definition 1. Clearly, $\left\langle X^{b}, N, L, H, S, r\right\rangle$ satisfies all the conditions in Definition 1 if in addition $r=H /(L+H)$.

Now, for any $r \in[0,1]$, let $X^{b}=D^{b}\left(t^{b}, r\right), x_{l}=s_{l}\left(t^{s}\right), x_{h}=s_{h}\left(t^{s}, X^{b}\right)$ and $N, L, H$, and $S$ be as in (5)-(8). Continuity of: $(i) D^{b}\left(t^{b}, r\right)$ in $r,(i i) s_{h}\left(t^{s}, X^{b}\right)$ in $X^{b}$ and (iii) F, implies 
that $H /(L+H)$ is continuous in $r$. Moreover, since $x_{h}>x_{l}$, then $L>0$, which implies that $0<H /(L+H)<1$. Since $H /(L+H)$ is continuous in $r$ and $0<H /(L+H)<1$, it follows by Brower's Fixed Point Theorem that for some $r \in[0,1], r=H /(L+H)$. Furthermore, since $0<H /(L+H)<1$, that value of $r \in(0,1)$.

Proof of Lemma 3. Suppose that $t_{*}^{s}, t_{*}^{b}$, and $r_{*}<1$ is a solution to the above problem. We use three necessary conditions for $t_{*}^{s}, t_{*}^{b}$, and $r_{*}<1$ to be an optimum and show that when $y$ is sufficiently large they cannot hold simultaneously.

1. $\partial \Pi\left(t_{*}^{s}, t_{*}^{b}, r_{*}\right) / \partial r \leq 0$. This is equivalent to $(E / m)\left(t_{*}^{s}+t_{*}^{b}\right) \frac{1-F\left(t_{*}^{s}\right)}{r_{*}}\left[g\left(t_{*}^{b}-y r_{*}\right) y-\frac{1-G\left(t_{*}^{b}-y r_{*}\right)}{r_{*}}\right] \leq$ 0 . Since $\left(t_{*}^{s}+t_{*}^{b}\right) \frac{1-F\left(t_{*}^{s}\right)}{r_{*}}>0$ (otherwise profit would be zero), this implies that

$$
y r_{*} \leq \frac{1-G\left(t_{*}^{b}-y r_{*}\right)}{g\left(t_{*}^{b}-y r_{*}\right)}
$$

2. $\partial \Pi\left(t_{*}^{s}, t_{*}^{b}, r_{*}\right) / \partial t^{b}=0$. Following the same steps as in 1 , we obtain that this condition implies that

$$
t_{*}^{s}+t_{*}^{b}=\frac{1-G\left(t_{*}^{b}-y r_{*}\right)}{g\left(t_{*}^{b}-y r_{*}\right)} .
$$

3. An increase $r$, combined with a decrease in $t^{s}$ such that seller participation $\left(1-F\left(t^{s}\right)\right) / r$ remains unchanged, cannot lead to an increase in profits. Given a change $d r$ in reputation, seller participation remains unchanged if the change in $t^{s}, d t^{s}$, satisfies: $d t^{s}=-\left(1 / r_{*}\right)[(1-$ $\left.\left.F\left(t_{*}^{s}\right)\right) / f\left(t_{*}^{s}\right)\right] d r$. Hence, the platform's profit does not increase with an increase in $r$ combined with such a change in $t^{s}$ iff $\partial \Pi\left(t_{*}^{s}, t_{*}^{b}, r_{*}\right) / \partial r-\left[\partial \Pi\left(t_{*}^{s}, t_{*}^{b}, r_{*}\right) / \partial t^{s}\right]\left(1 / r_{*}\right)[(1-$ $\left.\left.F\left(t_{*}^{s}\right)\right) / f\left(t_{*}^{s}\right)\right] \leq 0$. This condition implies that

$$
y r_{*} \leq \frac{1-F\left(t_{*}^{s}\right)}{f\left(t_{*}^{s}\right)}
$$

Note that if $r_{*}$ and $t_{*}^{s}$ satisfy the constraint of the problem, then the proposed change in 
$r$ and $t^{s}$ does not violate that constraint. To see this, note that $\partial\left\{\left[1-F\left(t_{*}^{s}\right)\right] /\left[1-F\left(t_{*}^{s}-\right.\right.\right.$ $d)]\} / \partial t^{s} \times d t^{s} / d r=1-\left\{f\left(t_{*}^{s}-d\right) /\left[1-F\left(t_{*}^{s}-d\right)\right]\right\} \times\left\{f\left(t_{*}^{s}\right) /\left[1-F\left(t_{*}^{s}\right)\right]\right\}<1$.

Next, we show that for $y$ sufficiently high, conditions (9)-(11) cannot hold simultaneously. Let $\underline{r}=\inf _{x \in \mathbb{R}}[1-F(x)] /[1-F(x-d)]$. We know it exists and that $\underline{r}>0$ since $[1-F(x)] /[1-$ $F(x-d)]$ is always non-negative and by assumption bounded away from zero. Take a value of $y$, say $\underline{y}$, such that $\underline{y} \underline{r} \geq[1-F(0)] / f(0)$ and $\underline{y} \underline{r} \geq[1-G(0)] / g(0)$. For any value of $y>\underline{y}$, $y r_{*}>\underline{y} \underline{r}$. Thus, from (9) and (11) it follows that $t_{*}^{b}-y r_{*}<0$ and $t_{*}^{s}<0$, since both $(1-G) / g$ and $(1-F) / f$ are decreasing by log-concavity of $g$ and $f$. Moreover, from (9) and (10), we obtain that $y r_{*} \leq t_{*}^{s}+t_{*}^{b}$. Clearly, conditions $t_{*}^{b}-y r_{*}<0, t_{*}^{s}<0$, and $y r_{*} \leq t_{*}^{s}+t_{*}^{b}$ cannot hold simultaneously.

Proof of Proposition 2. Analogous to that of Proposition 1 and therefore omitted.

Proof of Proposition 3. Consider an arbitrary pair of transaction fees $\left(t^{b}, t^{s}\right)$. Let $T^{s}$ be such that $s_{R}\left(t^{s}, T^{s}, D^{b}\left(t^{b}, 1\right)\right)=s_{h}\left(t^{s}, D^{b}\left(t^{b}, 1\right)\right)$. Denote that value of $T^{s}$ by $T_{0}^{s}$. Note that $T_{0}^{s}$ exists, since $(i) s_{R}\left(t^{s}, T^{s}, X^{b}\right)$ is continuous in $T^{s},(i i) s_{R}\left(t^{s}, 0, X^{b}\right)=s_{l}\left(t^{s}\right)<s_{h}\left(t^{s}, X^{b}\right)$ $\forall X^{b}>0$, and $\left(\right.$ iii) $\lim _{T^{s} \rightarrow+\infty} s_{R}\left(t^{s}, T^{s}, X^{b}\right)=+\infty \forall X^{b}>0$. Next, simply note that if fees are $t^{b}, t^{s}$ and $T_{0}^{s}$, then $r=1, X^{b}=D^{b}\left(t^{b}, 1\right), N=F\left(s_{R}\left(t^{s}, T^{s}, D^{b}\left(t^{b}, 1\right)\right)\right), L=0, H=1-N$, and $S=E / m$ satisfy all the conditions of Definition 2 .

Proof of Proposition 4. Suppose that the solution to the benchmark case is $\left\langle t_{0}^{s}, t_{0}^{b}, r=1\right\rangle$. Let $s_{0}$ denote the corresponding threshold type of seller above which sellers trade. Because $r=1$, only sellers who take the high action trade, which implies that $s_{0}=t_{0}^{s}$. Thus seller participation is given by $X_{0}^{s}=1-F\left(t_{0}^{s}\right)$ and in steady state there are $E / m$ sellers. Buyer participation is given by $X_{0}^{b}=1-G\left(t_{0}^{b}-y\right)$. The discounted value of the platform's profit associated with the solution of the benchmark case is $\Pi_{0}=(1 /(1-\delta)) X_{0}^{s} X_{0}^{b}\left(t_{0}^{b}+t_{0}^{s}\right) E / m$. 
Under moral hazard, this allocation can be implemented as follows. Choose the buyers' transaction fee $t_{1}^{b}=t_{0}^{b}$. Choose the sellers' transaction fee such that sellers of type above $s_{0}$ prefer to choose the high action. Using the no-cheating condition (4), this transaction fee, which we denote $t_{1}^{s}$, satisfies

$$
d=\alpha \frac{(1-m) \delta}{1-(1-m) \delta} X_{0}^{b}\left[s_{0}-t_{1}^{s}\right]
$$

and therefore $s_{h}\left(t^{s}, X_{0}^{b}\right)=s_{0}$. Now select the registration fee $T_{1}^{s}$ such that only sellers of type above $s_{0}$ trade. That is, choose

$$
T_{1}^{s}=V\left(s_{0}\right)=\frac{1}{1-(1-m) \delta} X_{0}^{b}\left[s_{0}-t_{1}^{s}\right]
$$

in which case only sellers of type above $s_{0}$ register (and trade). Thus, seller participation $X_{1}^{s}=X_{0}^{s}$. All those who trade choose the high action, which implies a reputation of one and a total number of sellers of $E / m$. Since $t_{1}^{b}=t_{0}^{b}$ buyer participation also equals the benchmark's: $X_{1}^{b}=X_{0}^{b}$. All that remains to show is that the platform's profit is the same as in the solution of the benchmark. It consists of the revenues associated with the transaction fees and the revenues associated with the registration fees. Specifically,

$$
\begin{aligned}
\Pi_{1} & =\frac{1}{1-\delta} X_{1}^{s} X_{1}^{b}\left(t_{1}^{b}+t_{1}^{s}\right) E / m+T_{1}^{s} X_{1}^{s} E / m+\sum_{\tau=1}^{\infty} m \delta^{\tau} T_{1}^{s} X_{1}^{s} E / m \\
& =\frac{1}{1-\delta} X_{0}^{s} X_{0}^{b}\left(t_{0}^{b}+t_{1}^{s}\right) E / m+T_{1}^{s} X_{0}^{s}\left(1+\frac{\delta m}{1-\delta}\right) E / m \\
& =\frac{1}{1-\delta} X_{0}^{s} X_{0}^{b}\left(t_{0}^{b}+t_{1}^{s}\right) E / m+T_{1}^{s} X_{0}^{s}\left(\frac{1-\delta(1-m)}{1-\delta}\right) E / m \\
& =\frac{1}{1-\delta} X_{0}^{s} X_{0}^{b}\left(t_{0}^{b}+t_{1}^{s}\right) E / m+\frac{1}{1-\delta(1-m)} X_{0}^{b}\left[s_{0}-t_{1}^{s}\right] X_{0}^{s}\left(\frac{1-\delta(1-m)}{1-\delta}\right) E / m \\
& =\frac{1}{1-\delta} X_{0}^{s} X_{0}^{b}\left(t_{0}^{b}+t_{0}^{s}\right) E / m \\
& =\Pi_{0}
\end{aligned}
$$


where the second equality follows from the fact that $X_{1}^{s}=X_{0}^{s}$ and $X_{1}^{b}=X_{0}^{b}$; the fourth equality follows by replacing $T_{1}^{s}$ with its value as given in (13) and noting that $s_{0}=t_{0}^{s}$.

\section{References}

[1] Armstrong, M., 2006, "Competition in Two-sided Markets," Rand Journal of Economics, $37,668-691$.

[2] Bolt, W. and A. Tieman, 2008, "Heavily Skewed Pricing in Two-Sided Markets," International Journal of Industrial Economics, 26, 1250-1255.

[3] Boudreau, K. and A. Hagiu, "Platform Rules: Multi-Sided Platforms As Regulators," forthcoming in A. Gawer, ed. Platforms, Markets and Innovation, Cheltenham, UK and Northampton, MA, US: Edward Elgar.

[4] Caillaud, B. and B. Jullien, 2003, "Chicken \& Egg: Competition Among Intermediation Service Providers," Rand Journal of Economics, 34, 309-328.

[5] Carrillo, J. D., 2000, "Corruption in Hierarchies," Annales d'Economie et de Statistique, $59,37-62$.

[6] Damiano, E. and H. Li, 2007, "Price Discrimination and Efficient Matching," Economic Theory, 30, 243-263.

[7] Hagiu, A., 2006, "Pricing and Commitment by Two-sided Platforms," Rand Journal of Economics, 37, 720-737.

[8] Hagiu, A., 2009, "Quality vs. Quantity and Exclusion by Two-sided Platforms," Harvard Business School Working Paper No.09-094, Cambridge, MA. 
[9] Hagiu, A. and B. Jullien, 2010, "Why do Intermediaries Divert Search?," Harvard Business School Working Paper No. 08-10, Cambridge, MA.

[10] Halaburda, H. and M. J. Piskorski, 2011, "Competing by Restricting Choice: The Case of Search Platforms," Harvard Business School Working Paper No.10-098, Cambridge, MA.

[11] Reisinger, M., 2010, "Unique Equilibrium in Two-Part Tariff Competition Between TwoSided Platforms. "Governance and the Efficiency of Economic Systems, Free University of Berlin, Humboldt University of Berlin, University of Bonn, University of Mannheim, University of Munich, Discussion Paper No. 308.

[12] Rochet, J.-C. and J. Tirole, 2003, "Platform Competition in Two-sided Markets," Journal of the European Economic Association, 1, 990-1029.

[13] Rochet, J.-C. and J. Tirole, 2006, "Two-sided Markets: A Progress Report," Rand Journal of Economics, 37, 645-667.

[14] Roger, G. and L. Vasconcelos, 2011, "Platform Pricing Structure and Moral Hazard," Australian Business School (University of New South Wales) Working Paper No. 2010-28, Sydney, Australia.

[15] Shapiro, C., 1983, "Premiums for High-Quality Products as a Return to Reputation," Quarterly Journal of Economics, 98, 659-679.

[16] Weyl, E.G., 2010, "A Price Theory of Multi-sided Platforms," American Economic Review, 100, 1642-1672. 